\title{
Co-creating an online TimeBank for inclusive research
}

\author{
Sarah Parsons ${ }^{1}$, Andy Power ${ }^{1}$, Melanie Nind $^{1}$, Ken Meacham ${ }^{2}$, Clare Hooper $^{2}$, \\ Anne Collis ${ }^{3}$ Mal Cansdale ${ }^{3}$, and Alan Armstrong ${ }^{4}$ \\ 1 University of Southampton, UK, \\ \{S.J.Parsons, a.power, m.a.nind\}@soton.ac.uk \\ 2 IT innovation, The University of Southampton, Southampton, UK, \\ \{kem, cjh\}@it-innovation.soton.ac.uk \\ 3 Barod, Community Interest Company, Bangor, UK \\ \{Anne, Mal, Alan\}@barod.org
}

\begin{abstract}
Participatory and inclusive approaches to research have become more common as researchers recognize the benefits of enabling the meaningful involvement of representative community users in the development of accessible technologies. One of the major challenges in this context is the how the involvement of community members can be appropriately supported in project-related activities: payment for time and contributions is a particularly difficult and longstanding issue. This paper discusses the inclusive development of an online Timebank involving community members with intellectual disabilities. The TimeBank is conceived as a tool that enables people to contribute their different expertise on the basis of time, rather than monetary reward. The development process of the TimeBank is described as well as the challenges faced by the research team. There is much potential in the Timebank idea, although considerable further research is needed to establish an accessible, usable, credible and trustworthy resource.
\end{abstract}

Keywords: Participatory research; User involvement; Intellectual disabilities; Equity; Inclusion

\section{Introduction}

Participatory design of technology encompasses a range of approaches to inclusive research that has developed within the field of human-computer interaction over the past 30 years [7]. Within this context and timeframe, there has also been increasing recognition of the value and importance of taking participatory and inclusive approaches to research in the intellectual disability field. While 'learning disability' is a preferred term in the UK, we use the more internationally recognised term 'intellectual disability', referring to a person's need for support in understanding complex information and/or communicating. This paper introduces an inclusively designed online TimeBank as a tool for (a) supporting the engagement and interaction of inclusive researchers, and (b) ensuring that 
time contributed to research is equitably recognized, valued and rewarded for inclusive researchers based at universities as well as for those based outside them e.g. in third sector and other community-based organisations.

Inclusive research is an umbrella term for methodological approaches that seek to provide greater sharing of power and decision-making in research [15]. Inclusive and participatory approaches share core principles through their focus:

"... on a process of sequential reflection and action, carried out with and by local people rather than on them. Local knowledge and perspectives are not only acknowledged but form the basis for research and planning" [8, p.1667].

The 'local people' and 'local knowledge' in this context refer to the expertise of community members who are not, or traditionally have not been, initiators of, or partners in, research i.e. those from outside academia who have tended to be marginalised from research or been the subjects of it [20]. Such inclusive research in the intellectual disability field has made important contributions to knowledge regarding the priorities and issues that matter in the lives of people with intellectual disabilities e.g. $[2,13]$, as well as empowering people through the development of skills, awareness, confidence, friendships and advocacy (e.g. $[3])$.

In a similar way, the participatory design of technologies has been identified as a key driver for ethically aware and appropriate development that seeks to ensure that technologies are usable and accessible to all [1]. Moreover, [4] suggest that the co-design and co-development of technologies with traditionally marginalised groups can promote digital empowerment and social inclusion, echoing the importance and strength of inclusivity that can be achieved by working 'with' others rather than researching 'on' others (cf. [8, 20]). Crucially, [1] argue that disabled people should be involved from the 'first steps' (p.486) of technology development in order to aim for more universal, accessible design.

However, there are challenges to participatory design research and inclusive research more broadly. Firstly, within inclusive research generally, there are important discussions about the extent to which all members of a research project can or should be included at all stages of the work, for example, including data analysis and interpretation (e.g. [14]). In participatory technology design, there are similar discussions that relate to the roles of community partners or intended 'end users' e.g. from tester and user, through to informant, and design partner [9], which have very different implications for the extent to which power and decision-making are shared. Indeed, [18] suggest that more equitable inclusive approaches to technology development may be especially challenging and thorny, not least because it may be unclear who the experts are regarding what a technology should be able to do. In other words, while inclusive research that focuses on the everyday lives of people with intellectual disabilities recognises and prioritises the expertise that individuals have on their own experiences, the same may not be true for technology development where a possible technological solution is novel and none of the individuals involved may be experts on all aspects of making the novel solution a reality [17]. 
Another major issue for inclusive and participatory research is how the involvement of community members can be appropriately supported in projectrelated activities. Payment for time and contributions is a particularly difficult and longstanding issue, both on a practical and ethical basis. Practically, payments for (often) unsalaried community users, or community users working in other jobs (such as schools), can be highly problematic because payments for research contributions may affect benefits and other income [12]. Ethically, if there is an aspiration to include community members from the very earliest stages of projects, including project planning and initial ideas, then there is a fundamental inequity between academic researchers who are usually salaried and paid for their time irrespective of whether project funding is in place vs. community members whose involvement is likely to be more dependent on securing project-specific funding [16].

Our proposed solution to this problem is to inclusively co-design an online TimeBank that enables university researchers and community members to be rewarded equitably for the time they contribute to inclusive research [11]. This paper outlines the process of initiation and development of the TimeBank idea, and discusses the extent to which co-design was achievable at all stages of the project. In other words, we document an inclusive research approach to solving an inclusive research problem, highlighting the challenges and opportunities for the design process and its outcome in the form of an online prototype.

\subsection{TimeBanking as a Solution to the Problem of Equity and Payment in Inclusive Research}

TimeBanks are 'community-based mutual volunteering schemes whereby participants give and receive services in exchange for time credits' [19, p.63], that is, time becomes currency [5]. The TimeBanking concept was originated in the US by Edgar Cahn who proposed that TimeBanks can enable democratic and reciprocal working partnerships, and the building of social capital, because people contribute their different expertise on the basis of time, rather than on the basis of any actual or perceived hierarchical monetary reward [6]. In this sense, a TimeBank is potentially a very inclusive device, enabling reciprocal and equitable exchanges for all participants [16].

TimeBanks have been developed to meet a diverse range of community-based needs in the UK and internationally $[5,10]$. While there seems to be increasing awareness and use of TimeBanks for supporting non-monetised exchanges [5] there has not yet, to the best of our knowledge, been a TimeBank targeted at inclusive researchers or developed through inclusive, participatory design processes. Beyond the core principles of instantiating equity and reciprocity of time, such a TimeBank could have numerous benefits, including:

- formalising and auditing currently invisible exchanges of time;

- enabling new and wider networks to be formed, moving beyond existing and local contacts; 
- improving the quality of research proposals and potential for wider impact through being able to involve the right people at the right time, particularly before and after grant-funded work;

- creating an inclusive space to encourage and enable more people with intellectual disabilities to express an interest in research.

\section{Methodology and Results: Co-designing the TimeBank}

Barod, a Community Interest Company run by people with and without intellectual disability, had the initial idea that TimeBanks could help inclusive research, and discussed the idea with university-based researchers. Internal funding was obtained for a small-scale project from the University of Southampton's Web Science Institute. Our 'core design' team (those who initially applied for the funding) therefore comprised three community-based inclusive researchers from Barod and four university-based researchers from different disciplines and areas of expertise including disability studies and web design. The project also involved a web developer who came on-board after initial scoping to help design and build the prototype. We called the project PRICE [Participation and Responsible Innovation in Co-Design for Exchange] and henceforth refer to the 'PRICE TimeBank'. Our methodology for developing the PRICE TimeBank comprised four main steps, each involving a number of activities, which are summarised next.

\subsection{Scoping and Agreeing the Initial Idea}

Each member of the team was tasked with searching for information to inform the development of the inclusive TimeBank, either from the academic research literature or from the web more broadly. Topics for searching included: examples of TimeBanking and accessible websites; history of the development of TimeBanks; web accessibility research; and digital tools for supporting and documenting communication and networking within the project. Our findings from these search exercises were shared with each other in a face-to-face meeting using accessible slides to show key ideas. We made plans at this meeting for the next stages of the project which were designed to inform the development of the online PRICE TimeBank prototype.

\subsection{Validating and Extending the Initial Idea}

We organised two main activities to further inform, validate and develop the PRICE TimeBank idea: (1) a face-to-face focus group and (2) a Twitter discussion. The focus group comprised 15 inclusive researchers in addition to members of the research team, specifically eight academic researchers and seven researchers with intellectual disabilities or autism. The Twitter discussion was a two-hour session in which contributors were able to tweet ideas and comments using a dedicated hashtag 'pricestudy'. There were 108 relevant contributions 
from 38 different Twitter accounts. Both activities contributed valuable ideas about how accessibility, online safety, trust, and reciprocity of the time exchanges could be managed, and reminded us of the need for caution in these areas. More information about the findings of these activities can be found in $[11,16]$.

\subsection{Development of the Idea into a Prototype}

Following the feedback from these activities, each core member of the design team submitted a 'walkthrough' describing the main features of the prototype that they felt were needed and how they would expect to be able to access, input and retrieve information from the PRICE TimeBank. These 'walkthroughs' were collated into an overall wish list which we negotiated with our web developer. The core design team provided feedback on specific features through a series of short iterative cycles based on visual mockups of the TimeBank over approximately two months, after which an early prototype of the online PRICE TimeBank was made available for preliminary evaluation.

\subsection{Evaluation and Feedback}

We approached seven people, already known to us, and asked them to try out the prototype individually; the seven people were: two inclusive researchers who had taken part in the focus group; two experts in web accessibility; one academic doing collaborative work in health; and two people with intellectual disabilities (one familiar with TimeBanks but not using the Web and one familiar with the Web but not TimeBanks). Each person was sent the link and asked to set up a user account, login, and explore the TimeBank. They were asked to feedback about current usability and accessibility, make suggestions for improvements, and comment on whether they felt the idea was understandable and useful.

Overall, feedback showed that these users liked the idea and felt there was a need for the TimeBank. There were many helpful suggestions for how the accessibility and usability of the TimeBank could be improved, for example by focusing on the overall layout in terms of text / visuals as well as the navigation between tabs and screens. There were also some suggestions for how the concept of the TimeBank might be conveyed more clearly, for example, it was felt that the TimeBank logo / image we used was not especially illuminating, and that we needed to be much clearer about what people with intellectual disabilities get back out of the Timebank. The use of specific examples and short videos was suggested as being potentially very helpful for providing this information.

\section{Outcomes and Contribution}

We have produced an early prototype of an online TimeBank for inclusive research and established, amongst members of the inclusive research community as well as the intellectual disability field more widely, that there is strong support for the idea. Current web accessibility guidelines do not provide information 
about how to make a website equally accessible to people with intellectual disabilities and academics, and we are in a good position to draft, test and refine guidelines as we further develop the PRICE TimeBank.

Nevertheless, there remained challenges about the extent to which all aspects of the design and development were fully inclusive. Our 'core design team' contributed equally to the initial scoping of the idea (Stage 1 above) and decisionmaking about the main aspects of the TimeBank (Stage 3). Thus, the core design team were design partners in the sense that $[9$, p.4] describes. i.e. '.. as equal stakeholders in the design of new technologies throughout the entire experience[who] contribute to the process in ways that are appropriate for [them] and the process'. Although Druin's work focused on children, her framework for thinking about the different roles of stakeholders in technology design processes can be applied much more widely, including people with intellectual disabilities. Indeed, she [9, p.19] emphasizes that different stakeholders can offer 'special experiences and viewpoints ... that other partners may not be capable of contributing'. This position is very much aligned with the fundamental ethos of inclusive research more broadly, as noted in the Introduction.

However, in terms of evaluation and further iterations outside our core design team, we were selective and targeted about whose views we sought at different stages. For example, in validating the idea (Stage 2) we invited members of our known networks to join discussions, either face-to-face or via Twitter. These participants were not design partners but were informants to the project which, according to [9], is when stakeholders are invited to contribute ideas before the technology is developed, often using low-tech methods to visualise concepts or ideas. We then involved other stakeholders as testers of the prototype TimeBank during the evaluation of the prototype (Stage 4). Testers usually evaluate prototypes before they are finalised and released for wider use and, therefore, can have quite immediate impacts on iterations as prototypes become more robust during further development [9]. However, only two out of the seven people involved at this stage had also contributed as informants via the focus group and so most knew very little about the project objectives.

There were some concerns within our core design team about the exclusion of many of our informants from the testing phase. This decision was made because it was felt the prototype was not yet accessible enough to be tested appropriately by all of the people who attended the focus group hence their invited involvement would have been excluding for them and, therefore, unethical. Nevertheless, although this decision was made for good pragmatic and ethical reasons, it was experienced as uncomfortable by the design team because questions arose about the extent to which we were able to be appropriately inclusive in all of our activities. This illustrates the specific challenge for technology development within inclusive and participatory research frameworks, as suggested by $[17,18]$. 


\section{Conclusions and Next Steps}

We have established an inclusive research partnership that works well, is trusting and open, and has produced a prototype online TimeBank for inclusive research. Despite all of our experiences in inclusive research, we were still challenged by some of the practical and ethical implications of co-design within technology development. We need to research, develop and test an improved version for the online TimeBank because, although we showed the concept works from our early version, we are painfully aware of its inadequacies. We also need to explore the concept of online trust, how to build trust and how to keep the TimeBank trustworthy in order to ensure that people's time and goodwill are not exploited. We intend to apply for further research funding to continue this work. It will be essential to maintain the strong involvement of people with intellectual disabilities in all of our plans and activities in order to ensure that issues of trust, relevance and credibility are appropriately investigated and addressed within future iterations of the TimeBank.

\section{Acknowledgements}

This project was funded by the WebScience Stimulus Fund at the University of Southampton, UK.

\section{References}

1. Abascal, J., \& Nicolle, C. (2005). Moving towards inclusive design guidelines for socially and ethically aware HCI. Interacting with Computers, 17(5), 484-505.

2. Abbott, S., \& McConkey, R. (2006). The barriers to social inclusion as perceived by people with intellectual disabilities. Journal of intellectual disabilities, 10(3), 275-287.

3. Atkinson, D. (2004). Research and empowerment: involving people with learning difficulties in oral and life history research. Disability \& Society, 19(7), 691-702.

4. Bleumers, L., All, A., Marin, I., Schurmans, D., Van Looy, J., Jacobs, A., Willaert, K. \& de Grove, F. (2012). State of Play of Digital Games for Empowerment and Inclusion: A Review of the Literature and Empirical Cases. EUR 25652 Joint Research Centre Institute for Prospective Technological Studies. Luxembourg: Publications Office of the European Union. doi:10.2791/36295

5. Bretherton, J. \& Pleace, N. (2014). An Evaluation of the Broadway Skills Exchange Time Bank. Centre for Housing Policy, University of York

6. Cahn, E. (2000) No More Throw-Away People: The Co-production Imperative. Washington, D.C: Essential Books

7. Coleman, R., Clarkson, J., Dong, H., Cassim, J. (2012). Design for Inclusivity: A Practical Guide to Accessible, Innovative and User-Centred Design. Gower Publishing Limited: Hampshire, UK.

8. Cornwall, A., \& Jewkes, R. 1995. What is participatory research? Social Science and Medicine 41(12): 1667-1676.

9. Druin, A. (2002). The role of children in the design of new technology. Behaviour and Information Technology, 21(1), 1-25. 
10. Glynos, J. \& Speed, E. (2012) Varieties of co-production in public services: time banks in a UK health policy context. Critical Policy Studies, 6(4), 402-433

11. Hooper, C., Nind, M., Parsons, S.J., Power, A. \& Collis, A. (2015) Building a Social Ma-chine: co-designing a TimeBank for inclusive research, WebSci '15, June 28 July 01, 2015, Oxford, UK. DOI: http://dx.doi.org/10.1145/2786451.2786472

12. Lewis, A., Parsons, S., Robertson, C., Feiler, A., Tarlton, B., Watson, D., \& Marvin, C. (2007) The role and working of reference, or advisory, groups involving disabled people: reviewing the experiences and implications of three contrasting research projects. British Journal of Special Education, 35(2), 78-84.

13. Manning, C. (2010). 'My memory's back!' Inclusive learning disability research using ethics, oral history and digital storytelling. British Journal of Learning Disabilities, 38(3), 160-167.

14. Nind, M. (2011). Participatory data analysis: a step too far? Qualitative Research, 11(4), 349-363.

15. Nind, M. (2014). What is Inclusive Research? Bloomsbury Academic: London

16. Nind, M., Armstrong, A., Cansdale, M., Collis, A., Hooper, C., Parsons, S. \& Power, A. (submitted). TimeBanking: Towards a co-produced solution for power and money issues in inclusive research.

17. Parsons, S. \& Cobb, S. (2013) Who chooses what I need? Child voice and user-involvement in the development of learning technologies for children with autism. EPSRC Observatory for Responsible Innovation in ICT. Available from http://observatory-rri.info/?q=obs-doc/report/case-study/473 [last accessed 22nd January 2016].

18. Parsons, S. \& Cobb, S. (2014) Reflections on the role of the 'users': challenges in a multi-disciplinary context of learner-centred design for children on the autism spectrum. International Journal of Research and Method in Education, 37(4), 421441.

19. Seyfang, G. (2004). Time banks: rewarding community self-help in the inner city. Community Development Journal 39(1), 62-71

20. Walmsley, J., \& Johnson, K. (2003). Inclusive research with people with learning disabilities: Past, present, and futures. Jessica Kingsley Publishers. 